PROCEEDINGS OF THE

AMERICAN MATHEMATICAL SOCIETY

Volume 138, Number 7, July 2010, Pages 2547-2552

S 0002-9939(10)10296-2

Article electronically published on March 3, 2010

\title{
ON IDENTITIES INVOLVING THE SIXTH ORDER MOCK THETA FUNCTIONS
}

\author{
JEREMY LOVEJOY
}

(Communicated by Ken Ono)

\begin{abstract}
We present $q$-series proofs of four identities involving sixth order mock theta functions from Ramanujan's lost notebook. We also show how Ramanujan's identities can be used to give a quick proof of four sixth order identities of Berndt and Chan.
\end{abstract}

\section{RAMANUJAN's SIXTH ORDER IDENTITIES}

The last four identities on p. 13 of Ramanujan's lost notebook [9] may be written as

$$
\begin{aligned}
q^{-1} \psi\left(q^{2}\right)+\rho(q) & =\left(-q ; q^{2}\right)_{\infty}^{2}\left(-q,-q^{5}, q^{6} ; q^{6}\right)_{\infty}, \\
\phi\left(q^{2}\right)+2 \sigma(q) & =\left(-q ; q^{2}\right)_{\infty}^{2}\left(-q^{3},-q^{3}, q^{6} ; q^{6}\right)_{\infty}, \\
2 \phi\left(q^{2}\right)-2 \mu(-q) & =\left(-q ; q^{2}\right)_{\infty}^{2}\left(-q^{3},-q^{3}, q^{6} ; q^{6}\right)_{\infty}, \\
2 q^{-1} \psi\left(q^{2}\right)+\lambda(-q) & =\left(-q ; q^{2}\right)_{\infty}^{2}\left(-q,-q^{5}, q^{6} ; q^{6}\right)_{\infty},
\end{aligned}
$$

where the "sixth order" mock theta functions $\phi, \psi, \rho, \sigma, \lambda$, and $\mu$ are defined by

$$
\begin{aligned}
\phi(q) & :=\sum_{n \geq 0} \frac{(-1)^{n} q^{n^{2}}\left(q ; q^{2}\right)_{n}}{(-q)_{2 n}} \\
\psi(q) & :=\sum_{n \geq 0} \frac{(-1)^{n} q^{(n+1)^{2}}\left(q ; q^{2}\right)_{n}}{(-q)_{2 n+1}} \\
\rho(q) & :=\sum_{n \geq 0} \frac{q^{n(n+1) / 2}(-q)_{n}}{\left(q ; q^{2}\right)_{n+1}} \\
\sigma(q) & :=\sum_{n \geq 0} \frac{q^{(n+1)(n+2) / 2}(-q)_{n}}{\left(q ; q^{2}\right)_{n+1}} \\
\lambda(q) & :=\sum_{n \geq 0} \frac{(-q)^{n}\left(q ; q^{2}\right)_{n}}{(-q)_{n}}, \\
\mu(q) & :=\sum_{n \geq 0} \frac{(-1)^{n}\left(q ; q^{2}\right)_{n}}{(-q)_{n}} .
\end{aligned}
$$

Received by the editors November 19, 2009.

2010 Mathematics Subject Classification. Primary 33D15.

(C)2010 American Mathematical Society Reverts to public domain 28 years from publication 
Here we use the usual $q$-series notation

$$
\left(a_{1}, a_{2}, \ldots, a_{k} ; q\right)_{n}:=\prod_{j=0}^{n-1}\left(1-a_{1} q^{j}\right)\left(1-a_{2} q^{j}\right) \cdots\left(1-a_{k} q^{j}\right),
$$

following the custom of dropping the "; $q$ " unless the base is something other than $q$. The convergence of $\mu(q)$ (as well as (1.7) below) is in the "Cesàro sense", which in this case means that the sum is obtained by averaging the limits of the even partial sums and the odd partial sums.

The identities (1.1) - (1.4) were proven by Andrews and Hickerson [3] by combining the Bailey pair method and the constant term method. The first point of this paper is that these identities follow immediately upon combining the $q$-series transformations

$$
\begin{aligned}
\frac{(-a q)_{\infty}}{(-q)_{\infty}} \sum_{n \geq 0} \frac{\left(x ; q^{2}\right)_{n}(a q)_{n}(-q / x)^{n}}{\left(q^{2} ; q^{2}\right)_{n}} & \\
= & \frac{\left(-a^{2} q / x ; q^{2}\right)_{\infty}}{\left(-q / x ; q^{2}\right)_{\infty}} \sum_{n \geq 0} \frac{\left(a^{2} q^{2} ; q^{2}\right)_{2 n}(-1)^{n} q^{2 n^{2}}}{\left(q^{4} ; q^{4}\right)_{n}\left(-a^{2} q / x ; q^{2}\right)_{2 n+1}} \\
& -\frac{a\left(-a^{2} q^{2} / x ; q^{2}\right)_{\infty}}{\left(-q^{2} / x ; q^{2}\right)_{\infty}} \sum_{n \geq 1} \frac{\left(a^{2} q^{2} ; q^{2}\right)_{n-1}(-q)^{n(n+1) / 2}}{(-q ;-q)_{n-1}\left(-a^{2} q^{2} / x ; q^{2}\right)_{n}}
\end{aligned}
$$

and

$$
\begin{aligned}
\frac{(-a q)_{\infty}}{(-q)_{\infty}} \sum_{n \geq 0} \frac{\left(x ; q^{2}\right)_{n}(a q)_{n}\left(-q^{2} / x\right)^{n}}{\left(q^{2} ; q^{2}\right)_{n}} & \\
= & \frac{\left(-a^{2} q^{2} / x ; q^{2}\right)_{\infty}}{\left(-q^{2} / x ; q^{2}\right)_{\infty}} \sum_{n \geq 0} \frac{\left(a^{2} q^{2} ; q^{2}\right)_{n}(-q)^{n(n+1) / 2}}{(-q ;-q)_{n}\left(-a^{2} q^{2} / x ; q^{2}\right)_{n+1}} \\
& +\frac{a\left(-a^{2} q^{3} / x ; q^{2}\right)_{\infty}}{\left(-q^{3} / x ; q^{2}\right)_{\infty}} \sum_{n \geq 0} \frac{\left(a^{2} q^{2} ; q^{2}\right)_{2 n}(-1)^{n} q^{2 n^{2}+4 n+1}}{\left(q^{4} ; q^{4}\right)_{n}\left(-a^{2} q^{3} / x ; q^{2}\right)_{2 n+1}}
\end{aligned}
$$

with the Rogers-Ramanujan type identities

$$
2 \sum_{n \geq 0} \frac{\left(q ; q^{2}\right)_{n}(-1)^{n}}{(q)_{n}}=\frac{\left(q ; q^{2}\right)_{\infty}\left(-q,-q^{2}, q^{3} ; q^{3}\right)_{\infty}}{\left(q^{2} ; q^{2}\right)_{\infty}}
$$

and

$$
\sum_{n \geq 0} \frac{\left(q ; q^{2}\right)_{n}(-q)^{n}}{(q)_{n}}=\frac{\left(q ; q^{2}\right)_{\infty}\left(-q^{3},-q^{3}, q^{3} ; q^{3}\right)_{\infty}}{\left(q^{2} ; q^{2}\right)_{\infty}} .
$$

Indeed, taking $x=q$ and $a= \pm 1$ in (1.5) and (1.6) and appealing to (1.7) and (1.8) we obtain

$$
\begin{aligned}
\mu(q) & =\frac{1}{2} \phi\left(q^{2}\right)-\sigma(-q), \\
\left(q ; q^{2}\right)_{\infty}^{3}\left(-q,-q^{2}, q^{3} ; q^{3}\right)_{\infty} & =\phi\left(q^{2}\right)+2 \sigma(-q), \\
\lambda(q) & =\rho(-q)+q^{-1} \psi\left(q^{2}\right), \\
\left(q ; q^{2}\right)_{\infty}^{3}\left(-q^{3},-q^{3}, q^{3} ; q^{3}\right)_{\infty} & =\rho(-q)-q^{-1} \psi\left(q^{2}\right),
\end{aligned}
$$

and this easily yields (1.1) - (1.4). 
The identities (1.7) and (1.8) come from letting $(a, b, c, d, e) \rightarrow(1, \infty, \infty,-\sqrt{q}, \sqrt{q})$ and $(q, \infty, \infty,-\sqrt{q}, \sqrt{q})$ in a limiting case of the Watson-Whipple transformation [6. p. 62, Ex. 2.22],

$$
\begin{aligned}
\sum_{n \geq 0} & \frac{\left(1-a q^{2 n}\right)(a, b, c, d, e)_{n}(-1)^{n} q^{n(n-1) / 2}(a q)^{2 n}}{(1-a)(q, a q / b, a q / c, a q / d, a q / e)_{n}(b c d e)^{n}} \\
& =\frac{(a q, a q / d e)_{\infty}}{(a q / d, a q / e)_{\infty}} \sum_{n \geq 0} \frac{(a q / b c, d, e)_{n}(a q / d e)^{n}}{(q, a q / b, a q / c)_{n}}
\end{aligned}
$$

and then applying the triple product identity [6, p. 357, Eq. (II.28)],

$$
\sum_{n \in \mathbb{Z}} z^{n} q^{n^{2}}=\left(-z q,-q / z, q^{2} ; q^{2}\right)_{\infty}
$$

As for (1.5) and (1.6), when $x \rightarrow \infty$ these are two entries from Ramanujan's lost notebook, proven by Andrews and Berndt [2, Entries (1.4.6) and (1.4.7)]. Their proof generalizes in a straightforward way to give (1.5) and (1.6) as follows.

Proof of (1.5) and (1.6). Two "Heine-type" transformations 1, Theorem $\mathrm{A}_{3}$ and Theorem $\mathrm{A}_{1}$ ] (or see [2, Theorem 1.21 and Theorem 1.22]) are required:

$$
\sum_{n \geq 0} \frac{\left(a ; q^{2}\right)_{n}(b)_{2 n} t^{n}}{\left(q^{2} ; q^{2}\right)_{n}(c)_{2 n}}=\frac{(b)_{\infty}\left(a t ; q^{2}\right)_{\infty}}{(c)_{\infty}\left(t ; q^{2}\right)_{\infty}} \sum_{n \geq 0} \frac{(c / b)_{n}\left(t ; q^{2}\right)_{n} b^{n}}{(q)_{n}\left(a t ; q^{2}\right)_{n}}
$$

and

$$
\begin{aligned}
\sum_{n \geq 0} \frac{\left(a ; q^{2}\right)_{n}(b)_{n} t^{n}}{\left(q^{2} ; q^{2}\right)_{n}(c)_{n}}= & \frac{(b)_{\infty}\left(a t ; q^{2}\right)_{\infty}}{(c)_{\infty}\left(t ; q^{2}\right)_{\infty}} \sum_{n \geq 0} \frac{(c / b)_{2 n}\left(t ; q^{2}\right)_{n} b^{2 n}}{(q)_{2 n}\left(a t ; q^{2}\right)_{n}} \\
& +\frac{(b)_{\infty}\left(a t q ; q^{2}\right)_{\infty}}{(c)_{\infty}\left(t q ; q^{2}\right)_{\infty}} \sum_{n \geq 0} \frac{(c / b)_{2 n+1}\left(t q ; q^{2}\right)_{n} b^{2 n+1}}{(q)_{2 n+1}\left(a t q ; q^{2}\right)_{n}}
\end{aligned}
$$

We begin with (1.5). First, setting $(q, a, b, c)=\left(q^{2}, q^{2} / t, a^{2} q^{2},-a^{2} q^{3} / x\right)$ and then letting $t \rightarrow 0$ in (1.9) we obtain

$$
\sum_{n \geq 0} \frac{\left(a^{2} q^{2} ; q^{2}\right)_{2 n}(-1)^{n} q^{2 n^{2}}}{\left(q^{4} ; q^{4}\right)_{n}\left(-a^{2} q / x ; q^{2}\right)_{2 n+1}}=\frac{\left(a^{2} q^{2} ; q^{2}\right)_{\infty}\left(q^{2} ; q^{4}\right)_{\infty}}{\left(-a^{2} q / x ; q^{2}\right)_{\infty}} \sum_{n \geq 0} \frac{\left(-q / x ; q^{2}\right)_{n} a^{2 n} q^{2 n}}{\left(q^{2} ; q^{2}\right)_{n}\left(q^{2} ; q^{4}\right)_{n}} .
$$

Next letting $(a, b, c, t) \rightarrow\left(-q^{2} / x, 0,-q^{2}, a^{2} q^{2}\right)$ and then setting $q=-q$ in (1.9) gives

$$
\begin{aligned}
& \sum_{n \geq 0} \frac{\left(-q^{2} / x ; q^{2}\right)_{n} a^{2 n+1} q^{2 n+1}}{(q)_{2 n+1}\left(-q^{2} ; q^{2}\right)_{n}} \\
& \quad=\frac{-a\left(-a^{2} q^{2} / x,-q ; q^{2}\right)_{\infty}}{\left(a^{2} q^{2} ; q^{2}\right)_{\infty}} \sum_{n \geq 1} \frac{\left(a^{2} q^{2} ; q^{2}\right)_{n-1}(-q)^{n(n+1) / 2}}{(-q ;-q)_{n-1}\left(-a^{2} q^{2} / x ; q^{2}\right)_{n}}
\end{aligned}
$$


Then letting $(a, b, c, t) \rightarrow(x, a q, 0,-q / x)$ in (1.10) and multiplying both sides by $(-a q)_{\infty} /(-q)_{\infty}$, we have

$$
\begin{aligned}
\frac{(-a q)_{\infty}}{(-q)_{\infty}} \sum_{n \geq 0} \frac{\left(x ; q^{2}\right)_{n}(a q)_{n}(-q / x)^{n}}{\left(q^{2} ; q^{2}\right)_{n}} & \\
= & \frac{\left(a^{2} q^{2} ; q^{2}\right)_{\infty}}{\left(-q^{2},-q / x ; q^{2}\right)_{\infty}} \sum_{n \geq 0} \frac{\left(-q / x ; q^{2}\right)_{n} a^{2 n} q^{2 n}}{(q)_{2 n}\left(-q ; q^{2}\right)_{n}} \\
& +\frac{\left(a^{2} q^{2} ; q^{2}\right)_{\infty}}{\left(-q,-q^{2} / x ; q^{2}\right)_{\infty}} \sum_{n \geq 0} \frac{\left(-q^{2} / x ; q^{2}\right)_{n} a^{2 n+1} q^{2 n+1}}{(q)_{2 n+1}\left(-q^{2} ; q^{2}\right)_{n}} .
\end{aligned}
$$

Finally, applying (1.11) and (1.12) to the first and second terms on the right-hand side of (1.13) gives (1.5).

The proof of (1.6) is similar so we just sketch it. We let $(a, b, c, t) \rightarrow$ $\left(x, a q, 0,-q^{2} / x\right)$ in (1.10), multiply both sides by $(-a q)_{\infty} /(-q)_{\infty}$, then transform the first term on the right-hand side using the result of letting $(a, b, c, t) \rightarrow$ $\left(-q^{2} / x, 0,-q, a^{2} q^{2}\right)$ and then setting $q=-q$ in (1.9), and transform the second term on the right-hand side using the result of setting $(q, b, c, t)=\left(q^{2}, a^{2} q^{2},-a^{2} q^{5} / x, q^{6} / a\right)$ and letting $a \rightarrow 0$ in (1.9).

\section{Berndt And Chan's SiXth ORDER IDEntities}

Recently Berndt and Chan [4 defined two more sixth order mock theta functions:

$$
\begin{aligned}
\phi_{-}(q) & :=\sum_{n \geq 1} \frac{(-q)_{2 n-1} q^{n}}{\left(q ; q^{2}\right)_{n}}, \\
\psi_{-}(q) & :=\sum_{n \geq 1} \frac{(-q)_{2 n-2} q^{n}}{\left(q ; q^{2}\right)_{n}} .
\end{aligned}
$$

Using the same methods as Andrews and Hickerson, they proved the four identities

$$
\begin{aligned}
-2 q^{-1} \psi_{-}\left(q^{2}\right)+\rho(q) & =\left(-q^{2} ; q^{2}\right)_{\infty}^{3}\left(q^{6}, q^{6}, q^{12} ; q^{12}\right)_{\infty} \\
-\phi_{-}\left(q^{2}\right)+\sigma(q) & =q\left(-q^{2} ; q^{2}\right)_{\infty}^{2}\left(-q^{6},-q^{6}, q^{6} ; q^{6}\right)_{\infty} \\
4 \phi_{-}\left(q^{2}\right)+2 \mu(q) & =\left(-q ; q^{2}\right)_{\infty}^{2}\left(-q^{3},-q^{3}, q^{6} ; q^{6}\right)_{\infty} \\
4 q^{-1} \psi_{-}\left(q^{2}\right)+\lambda(q) & =\left(-q ; q^{2}\right)_{\infty}^{3}\left(q^{3}, q^{9}, q^{12} ; q^{12}\right)_{\infty}
\end{aligned}
$$

The second point of this paper is that the identities of Berndt and Chan follow readily from those of Ramanujan. Indeed, using equations (1.1) - (1.4) to eliminate $\rho, \sigma, \mu$, and $\lambda$ from equations (2.1) - (2.4), we have the equivalent identities

$$
\begin{aligned}
& q^{-1} \psi\left(q^{2}\right)+2 q^{-1} \psi_{-}\left(q^{2}\right) \\
& \quad=\left(-q ; q^{2}\right)_{\infty}^{2}\left(-q,-q^{5}, q^{6} ; q^{6}\right)_{\infty}-\left(-q^{2} ; q^{2}\right)_{\infty}^{3}\left(q^{6}, q^{6}, q^{12} ; q^{12}\right)_{\infty}, \\
& \begin{aligned}
\phi\left(q^{2}\right) & +2 \phi_{-}\left(q^{2}\right) \\
\quad & \left(-q ; q^{2}\right)_{\infty}^{2}\left(-q^{3},-q^{3}, q^{6} ; q^{6}\right)_{\infty}-2 q\left(-q^{2} ; q^{2}\right)_{\infty}^{2}\left(-q^{6},-q^{6}, q^{6} ; q^{6}\right)_{\infty} \\
2 \phi\left(q^{2}\right) & +4 \phi_{-}\left(q^{2}\right) \\
\quad & \left(-q ; q^{2}\right)_{\infty}^{2}\left(-q^{3},-q^{3}, q^{6} ; q^{6}\right)_{\infty}+\left(q ; q^{2}\right)_{\infty}^{2}\left(q^{3}, q^{3}, q^{6} ; q^{6}\right)_{\infty}, \\
2 q^{-1} & \psi\left(q^{2}\right)+4 q^{-1} \psi_{-}\left(q^{2}\right) \\
\quad & =\left(-q ; q^{2}\right)_{\infty}^{2}\left(-q,-q^{5}, q^{6} ; q^{6}\right)_{\infty}-\left(q ; q^{2}\right)_{\infty}^{3}\left(-q^{3},-q^{9}, q^{12} ; q^{12}\right)_{\infty} .
\end{aligned}
\end{aligned}
$$


The modularity of the left-hand side in the case of $\phi+2 \phi_{-}$follows from an identity of Ramanujan [2, Eq. (3.4.2)],

$$
\begin{gathered}
\sum_{n \geq 0} \frac{(-1)^{n}\left(q ; q^{2}\right)_{n} q^{n^{2}}}{(-q)_{2 n}}+2 \sum_{n \geq 1} \frac{(-q)_{2 n-1} q^{n}}{\left(q ; q^{2}\right)_{n}} \\
\quad=\frac{1}{(q)_{\infty}}\left(1+6 \sum_{n \geq 0}\left(\frac{q^{6 n+2}}{1-q^{6 n+2}}-\frac{q^{6 n+4}}{1-q^{6 n+4}}\right)\right),
\end{gathered}
$$

combined with an identity of Lorenz [7] (or see [5]),

$$
\sum_{m, n \in \mathbb{Z}} q^{n^{2}+m n+m^{2}}=1+6 \sum_{n \geq 0}\left(\frac{q^{3 n+1}}{1-q^{3 n+1}}-\frac{q^{3 n+2}}{1-q^{3 n+2}}\right)
$$

while the modularity of $\psi+2 \psi$ - follows from the case $a \rightarrow-1$ of another identity of Ramanujan [2, Eq. (3.4.6)],

$$
\begin{aligned}
& \sum_{n \geq 0} \frac{\left(-a q ; q^{2}\right)_{n} a^{n+1} q^{(n+1)^{2}}}{(a q)_{2 n+1}}-\sum_{n \geq 1} \frac{(1 / a)_{2 n-1} q^{n}}{\left(-q / a ; q^{2}\right)_{n}} \\
& \quad=\frac{q\left(q^{2} ; q^{2}\right)_{\infty}\left(-a^{3},-q^{6} / a^{3}, q^{6} ; q^{6}\right)_{\infty}}{a(a q)_{\infty}\left(q ; q^{2}\right)_{\infty}\left(-a,-q^{2} / a,-q / a, q^{2} ; q^{2}\right)_{\infty}} .
\end{aligned}
$$

Now (2.5) - (2.8) follow from standard computational techniques for modular forms. For example, using (2.9) in (2.5), setting $q=-q$, and doing a little rearranging we have that (2.5) is equivalent to

$$
\frac{-3 \eta^{4}(24 z)}{\eta^{2}(12 z)}=\frac{\eta(8 z) \eta^{3}(2 z) \eta(24 z)}{\eta(6 z) \eta^{2}(4 z)}-\frac{\eta^{4}(8 z)}{\eta^{2}(4 z)}
$$

where $\eta(z):=q^{1 / 24}(q)_{\infty}$ and $q:=e^{2 \pi i z}$. This is an equality between holomorphic modular forms of weight 1 on $\Gamma_{0}(24)$ (with a certain character), so its truth is established by verifying that the $q$-expansions of both sides agree up to $q^{4}$. (Those unfamiliar with this method might consult [8].)

\section{REFERENCES}

[1] G.E. Andrews, On basic hypergeometric series, mock theta functions, and partitions. I, Quart. J. Math. 17 (1966), 64-80. MR.0193282 (33:1502)

[2] G.E. Andrews and B.C. Berndt, Ramanujan's Lost Notebook. Part II, Springer, New York, 2009. MR 2474043

[3] G.E. Andrews and D. Hickerson, Ramanujan's "lost" notebook. VII: The sixth order mock theta functions, Adv. Math. 89 (1991), 60-105. MR.1123099 (92i:11027)

[4] B.C. Berndt and S.H. Chan, Sixth order mock theta functions, Adv. Math. 216 (2007), 771786. MR2351377 (2008i:33046)

[5] J.M. Borwein, P.B. Borwein, and F.G. Garvan, Cubic modular identities of Ramanujan, Trans. Amer. Math. Soc. 343 (1994), 35-47. MR1243610 (94j:11019)

[6] G. Gasper and M. Rahman, Basic Hypergeometric Series, 2nd Ed., Cambridge Univ. Press, Cambridge, 2004. MR2128719 (2006d:33028)

[7] L. Lorenz, Bidrag til tallenes theori, Tidsskrift for Mathematik (3) 1 (1871), 97-114. 
[8] K. Ono, The Web of Modularity: Arithmetic of the Coefficients of Modular Forms and $q$-series, CBMS Regional Conference Series in Mathematics, 102, American Mathematical Society, Providence, RI, 2004. MR2020489 (2005c:11053)

[9] S. Ramanujan, The Lost Notebook and Other Unpublished Papers, Narosa Publishing House, New Delhi, 1988. MR947735 (89j:01078)

Laboratorie d'Informatique Algorithmique: Fondements et Applications, CNRS UMR 7089, Université Denis Diderot - Paris 7, Case 7014, 75205 Paris Cedex 13, France

E-mail address: lovejoy@liafa.jussieu.fr 